\title{
Effectiveness of a tele-rehabilitation intervention to improve performance and reduce morbidity for people post hip fracture - study protocol for a randomized controlled trial
}

Yafit Gilboa ${ }^{1 *}$ (D), Talia Maeir ${ }^{1}$, Sharon Karni ${ }^{2}$, Michal E. Eisenberg ${ }^{2}$, Meir Liebergall ${ }^{3}$, Isabella Schwartz ${ }^{3}$ and Yakir Kaufman ${ }^{2}$

\begin{abstract}
Background: Most surviving hip-fracture patients experience reduced mobility and lose some of their functional ability, which increases the risk of complications and rehospitalization. Post-discharge transitional programs to reduce readmissions and disabilities have shown some success. Telerehabilitation refers to the use of technologies to provide rehabilitation services to people in their homes. Considering the need for long-term follow-up care for people with hip fracture, in-home telerehabilitation could increase independence, decrease hospital stays and reduce the burden for caregivers. The objective of this study is to investigate the effectiveness of an intervention program based on telerehabilitation on activities of daily living (ADL), quality of life (QOL), depression and burden on caregivers compared to face-to-face home visits and usual care of community-dwelling older adults after hip fracture.

Methods/design: This will be a three-armed randomized control trial (RCT) including pre/post intervention and followup. The trial will include 90 older people with hip fractures who will be randomly assigned to a telerehabilitation group ( $N=30)$, face-to-face visits $(N=30)$ and a control group. The aim of the intervention is to improve the transition from rehabilitation units to community dwelling. It will include 10 videoconferencing/ face-to-face sessions from an occupational therapist in the presence of the primary caregiver. Each session will be utilized to guide the participants to achieve their self-identified goals, focusing on problem-solving for daily life situations and on the ability to implement the discussed strategies for a variety of activities. Outcome measures include Functional Independence Measure (FIM) for evaluation of ADL, SF-12 for evaluation of Health-related QOL, The Geriatric Depression Scale (GDS) and The Zarit Caregiver Burden Scale. Data will be analyzed using Repeated measures MANOVA.
\end{abstract}

Discussion: The current study will enable the cost-effectiveness examination of a suggested rehabilitation service based on available technology. The proposed intervention will increase accessibility of in-home rehabilitation services, improve function and health, and reduce economic burden.

Trial registration: NCT03376750 (12/15/2017).

Keywords: Hip fracture, Elderly, Tele-rehabilitation, Cost-effectiveness, In-home rehabilitation, Transition, Occupational therapy, Community dwelling, Problem-solving

\footnotetext{
*Correspondence: Yafit.gilboa@mail.huji.ac.il

'School of Occupational Therapy, Faculty of Medicine, Hadassah and the

Hebrew University of Jerusalem, Mount Scopus, 91240 Jerusalem, Israel

Full list of author information is available at the end of the article
}

(c) The Author(s). 2019 Open Access This article is distributed under the terms of the Creative Commons Attribution 4.0 International License (http://creativecommons.org/licenses/by/4.0/), which permits unrestricted use, distribution, and reproduction in any medium, provided you give appropriate credit to the original author(s) and the source, provide a link to the Creative Commons license, and indicate if changes were made. The Creative Commons Public Domain Dedication waiver (http://creativecommons.org/publicdomain/zero/1.0/) applies to the data made available in this article, unless otherwise stated. 


\section{Background}

Hip fracture is one of the most serious osteoporotic fracture, and the second most common cause of hospitalization among the age group older than 65 years [1]. Hip fractures occur predominantly in the geriatric population [2, 3], due to higher rates of osteoporosis and falls [4]. The lifetime risk of hip fracture is $9 \%$ for women and $4 \%$ for men [5]. Hip fractures are a major public health problem in terms of patient morbidity, mortality and costs to health and social care $[4,6]$. With an ageing population, and a growing proportion of people aged 80 years or older, the societal and economic burden of osteoporosis-related fractures is deemed to be increasing [7]. The healthcare costs for hip fractures are the highest (€20,000/patient) in comparison with other costs of injuries [8]. Osteoporotic hip fractures incur a high economic cost mainly due to the first hospitalization [8], but also due to subsequent outpatient visits, home care [6] and complications following initial hospital discharge [4, 9]. Therefore, further research is needed to identify the most effective and cost-effective rehabilitation pathways for these patients [10].

A third of older adults with hip fracture will be readmitted to hospital within 30 days of their return home [11], and up to $20 \%$ of patients die in the first year following hip fracture [12]. Hip fractures have profound effects on quality of life (QoL) and activities of daily living (ADL) $[13,14]$. Most patients surviving hip fracture experience reduced mobility, lose their functional ability [15] and independence [16], and suffer from depression [17]. A systematic review found that after 1 year, $29 \%$ of hip-fracture patients did not reach their pre-fracture level of ADL, and $35 \%$ were not able to walk independently [2].

Hip fractures nearly always necessitate hospitalization and rehabilitation $[18,19]$. Rehabilitation is a significant factor impacting the level of functional independence following hip fracture [20]. In the United States, more than 90\% of Medicare beneficiaries utilize post-acute-care rehabilitation after an acute hip fracture hospitalization [21]. In Israel, $73.5 \%$ of the hip-fracture patients received inpatient rehabilitation, $14.3 \%$ received ambulatory rehabilitation treatment without an inpatient phase, and $12.2 \%$ received no rehabilitation [22].

The objective of rehabilitation is successful community discharge - facilitating a safe return to the community, thereby minimizing the likelihood of long-term institutionalization or reentry into the healthcare system [21]. However, a substantial decline in functional ability of hip-fracture patients occurs 1 year post discharge from rehabilitation. More than half of the hip-fractured elderly patients were unable to maintain their rehabilitation achievements 1 year post discharge [23]. Thus, to be efficacious in reducing or reversing disability after hip fracture, rehabilitation needs to be individualized, include many components, be progressive, and span a sufficiently long period [24].

Transitions between healthcare settings are a high-risk period for care quality and patient safety, mainly for older patients - such as those with hip fracture - who have complex needs and may undergo multiple care transitions [25]. Particularly for older adults, transitions are often plagued with discontinuity and a lack of coordination [26]. Patterns and problems associated with transitions after hip fracture in older adults include weight loss, delirium, depression, pressure ulcers, falls, and urinary incontinence [27], resulting in increased use of hospital, ambulatory and emergency services [26]. Gaps in communication and timely delivery of information are commonly cited barriers for inter-professional teams working along the continuum of care [25]. Strategies to improve transitional care to older hip-fracture patients should include improved patient and family involvement at the time of transition [27], communication and information sharing [25].

To summarize, hip fractures are one of the most common and potentially devastating injuries amongst the geriatric population [1]. The incidence, morbidity, and medical costs associated with hip fracture among the elderly are well-recognized [20]. The economic burden associated with hip fractures calls for the investigation of innovative and cost-effective forms of services [28]. Healthcare providers should be aware of the necessity for an individualized, holistic, long-term post-rehabilitation intervention program to prevent functional decline $[9,23]$.

Traditional in-home therapy provides the opportunity for rehabilitation intervention to occur within the context of everyday task performance [29]. Moreover, home rehabilitation programs have positive long-term effects in comparison with conventional care after hip fracture in elderly people [30-33]. Nonetheless, although in-home rehabilitation has been generally successful in preventing ADL decline [34] and facilitating recovery of ADL functioning and locomotion [35], problems caused by distance, the cost of travel and absence of healthcare personnel limit its utility [36]. To overcome these limitations, several studies have demonstrated that in-home therapy delivered to the elderly using interactive video-conferencing can successfully treat ADL task-performance deficits [37-39]. These studies suggest that relatively inexpensive video-conferencing technology can maintain the patient-therapist interactions, and provide a pragmatic alternative to face-to- face home visits by a therapist for improving function [36] and reducing healthcare spending [39].

This "telerehabilitation" strategy for chronic and prolonged disease management involves the patient in the process of medical care, provides continuous medical monitoring and early symptoms detection allows the ability to promptly respond to an acute exacerbation [40]. In addition, it can help to lower the psychological threshold 
for accessing medical care in people who are not aware of the seriousness of their symptoms, or who might otherwise be reluctant to access face-to-face care [41]. The evolving telecommunications industry has been proposed as a realistic solution to reduce healthcare cost, time, hospital visits [42] and caregiver burden [43], and provide long-term and easily accessible remote medical services [39, 44]. By using telerehabilitation services, client access to care can be improved and the reach of clinicians expanded, thus enhancing the continuity of care to persons with disabling conditions $[45,46]$. A study that compared the effectiveness of video-conferencing with traditional outpatient clinic visits in the implementation of the management plan showed that video-conferencing is a valid alternative for orthopedic specialist consultations [47]. The results of another study suggest that conducting pre-admission occupational therapy "home visits" via the Internet with patients who are scheduled to undergo a total hip or knee replacement is both feasible and accurate [48].

Recent studies demonstrated the potential of home-based telerehabilitation in older adults and individuals with mobility impairment $[49,50]$. As far as we know, however, only one paper so far has aimed to assess the impact of an older patient's acceptance of hip-fracture telerehabilitation. They found that this intervention protocol targeting individualized impairments was feasible, safe and effective for use in home-based rehabilitation of older adults after hip fracture [51]. However, this research included only 10 patients and employed a quasi-experimental pre/post design without control group or follow-up. A study with longer duration and larger sample size, which also includes follow-up, needs to be done to measure hip-fracture recovery outcomes including functional parameters.

Some papers have addressed the issue of cost-effectiveness of telerehabilitation services to older adults. The first research assessed the feasibility of providing home-based telerehabilitation as an alternative to traditional ambulatory rehabilitation in patients post stroke, fracture or long hospital admission. There was a 50\% reduction in staff's home visits due to the telerehabilitation intervention. The therapists were able to provide twice the amount of service and direct patient-contact time, whilst reducing by $50 \%$ time they spent travelling [52]. In addition, the effectiveness of a virtual reality-based telerehabilitation program for balance recovery after stroke was compared with conventional physical therapy program in the clinic. Results showed that the clinic-based intervention had a higher cost relative to the virtual reality intervention [53]. An Israeli case study described novel integrated motor imagery treatment designed for the rehabilitation of walking, which was delivered in the home through telerehabilitation for a person after stroke. Compared with on-site delivery, the telerehabilitation sessions resulted in lower therapist travel time and cost, as well as shorter therapy sessions [54]. Economic analyses were conducted as part of trials using telerehabilitation for total knee arthroplasty $[55,56]$. The cost for a single session of in-home telerehabilitation compared to conventional home-visit rehabilitation was lower or about the same, depending on the distance between the patient's home and healthcare center. A favorable cost differential was observed when the patient was more than $30 \mathrm{~km}$ from the provider [56]. Even if transportation costs were excluded, telerehabilitation was still a cost-effective alternative to usual care [55].

To summarize, the idea of telerehabilitation is becoming a more concrete means to deliver services to patients in their home environment, allowing patients to gain self-confidence in their ability to manage their health conditions [46]. Recent studies demonstrated the potential of home-based telerehabilitation in older adults $[47,49,50]$ and its cost-effectiveness [52, 53]. However, limited evidence exists on the feasibility of home-based telerehabilitation in older adults after hip fracture.

\section{Methods \\ Study aims}

The primary aim of this study is to compare the effectiveness of in-home telerehabilitation and face-to-face home visit intervention programs, as opposed to the standard care currently offered to community-dwelling older adults after hip fracture. The secondary aims of this study are: (1) to evaluate the maintenance of the interventions' accomplishments 3 months after completion; (2) to estimate the cost-effectiveness of the intervention programs; and (3) to understand the user experience of the patients and caregivers.

\section{Trial design, randomization and recruitment}

This study is a three-armed, evaluator-blind, randomized control trial (RCT) and will be implemented according to CONSORT guidelines [57]. Participants will be assigned to research and control groups by using a blocked randomized controlled design, stratified based on age. The randomization sequence will be computer generated and will be known only to the responsible investigator.

All participants will be recruited at the time of discharge from hospital. Health professionals employed at the hospital will screen and refer to a research assistant the interested clients who meet the inclusion criteria. The research assistant will obtain informed consent from caregivers and patients prior to randomization and trial inclusion. Participants $(n=90)$ will be randomized after baseline assessment in the three-arm study. The rehabilitation program will be identical in both intervention groups; only the delivery mode will differ: in-home telerehabilitation or face-to-face home visit. The patients allocated to all groups will continue to receive the standard usual care to improve adherence to intervention protocols. All outcomes will be 


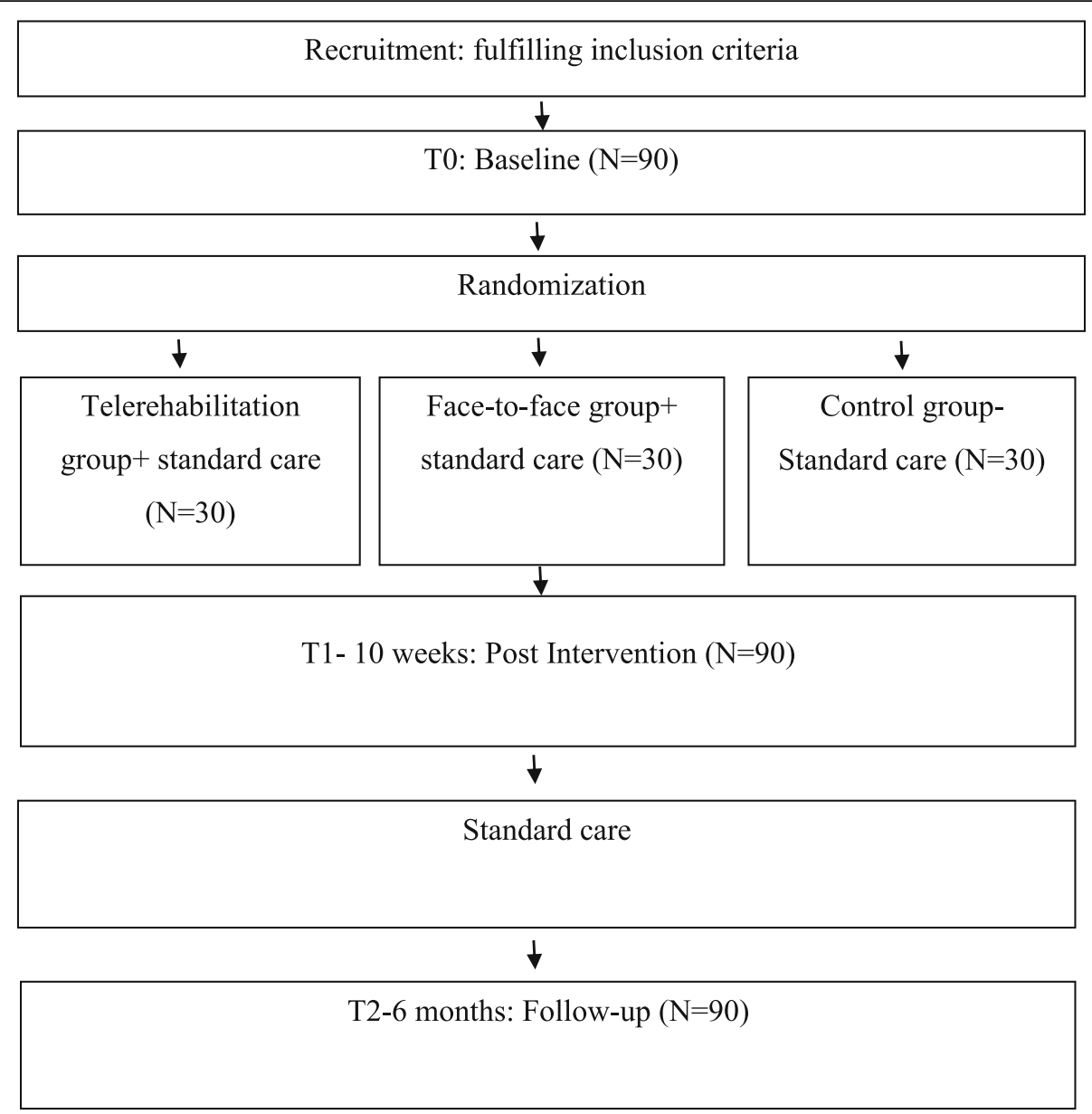

Fig. 1 Schematic illustration of the study design

measured at all three time points: Baseline-pretest (T0), immediately following intervention (post intervention 10 weeks) (T1), and a 6-month follow-up (T2) (See Fig. 1).

Recruitment and baseline testing will be carried out at Herzog and Hadassah Medical Centers, Jerusalem Israel. Intervention sessions will be conducted by video-conferencing or face-to-face visit while the participants are at their homes. Post-intervention and follow-up assessments will be carried out by e-mail or phone. The participation in the study will discontinue upon participants' request.

\section{Study population}

Participants in post-acute phases of recovery after hip fracture (ICD-10 code: S72.0) [58].

\section{Inclusion criteria}

Older adults (age $\geq 60$ years) after hip fracture, who will be discharged from the rehabilitation or orthopedics units of Herzog and Hadassah Hospitals, Jerusalem, between January 2017 and December 2019. Discharge will be from rehabilitation to a non-institutionalized setting. Patients with broadband or Wi-Fi in their home and able to operate an iPad or computer independently. Functional Independence Measure [49] score at rehabilitation discharge: FIM > 90. Every participant will designate a caregiver (a close friend, family member, or support worker) aged over 18, who will agree to cooperate in the research and be present during the interventional phases to promote participants' retention.

\section{Exclusion criteria}

Aphasia, cognitive impairment (Montreal Cognitive Assessment scores: MOCA<21) [59], other degenerative neurological diagnoses, current major depressive or psychotic disorder, or other acute or chronic health condition that will influence their ability to participate in the study.

\section{Intervention}

The aim of the intervention is to improve functional outcomes during the transition from rehabilitation units to community living and facilitate involvement in life roles in the community. The intervention program will be based on the Cognitive Orientation to daily 
Occupational Performance (CO-OP) [60]. CO-OP reflects a paradigm shift from component-focused rehabilitation traditionally used, to a broader, client centered, occupation based approach. CO-OP has the potential to support the long-term maintenance of therapeutic achievements following a rehabilitation and encourage the generalization and transfer of skills that will likely lead to continued improvement, rather than additional deterioration [61]. The strategy of $\mathrm{CO}-\mathrm{OP}$ is to focus treatments directly on improving performance in everyday life activity, rather than treating the underlying impairments and hoping for secondary improvement in meaningful activities [62]. The consensus of the literature examining the CO-OP approach suggests that its use in many novel populations and settings is promising and is expanding [63].

CO-OP is a top-down, task-oriented, client-centered approach that uses an iterative process of dynamic performance analysis and guided discovery to enable individuals to identify strategies that will improve performance [63]. Studies that implement these principal elements in their intervention program described supportive results among elderly after hip fracture [30, 33, 64, 65]. The findings indicate that participating in treatment which focuses on individually chosen and personally meaningful activities improves quality of life [33], balance confidence, independence and physical activity [64] with long-term effect [30] and decreased length of hospital stays [65]. Additionally, previous research demonstrated the effectiveness of $\mathrm{CO}-\mathrm{OP}$ on function-related goals in post-stroke elderly patients $[61,62,66-68]$, which suggests that $\mathrm{CO}-\mathrm{OP}$ may have a broader positive effect on recovery than conventional occupational therapy care [61, 62]. The older adult populations were comprised of those with no diagnosis of dementia, depression, or cognitive impairment upon testing $[62,63]$.

In line with previous protocol that included post-stroke elderly patients with a parallel cognitive profile [62], participants will be receiving $10 \mathrm{~h}$ of training (1-h sessions/week) from a trained occupational therapist. Through dialogues, the therapist directs the individual to discuss the task's purpose (goal), identify strategies to achieve it (plan), execute these strategies (do), and evaluate their progress and outcome (check). The intervention will be performed by real-time video-conferencing using an $\mathrm{iPad}^{\circ}$ and Skype ${ }^{\mathrm{Tm}}$, a software program that allows video calls over the Internet. The CO-OP approach administered in a telerehabilitation format was found to be feasible for adults with traumatic brain injury [69].

\section{Treatment fidelity}

The occupational therapists will record digital audio or take notes each session. The data of the process and content of the intervention will be used to ensure the treatment fidelity of the intervention. Recordings of the first five sessions of the first 5 participants will be monitored. The occupational therapists providing the intervention and an additional research assistant will review the session recordings to ensure that the $\mathrm{CO}-\mathrm{OP}$ is delivered as intended, following the essential aspects of intervention.

\section{Control group}

The purpose of the control group is to control for changes that may occur with the passage of time in the estimate of the efficacy of the intervention [70]. This group will receive the usual care and rehabilitation by the common routine for patients with hip fracture, and will be discharged with no further therapy as part of the study. In addition, the clinicians will receive no direction from the research study staff.

\section{Measurements \\ Sample characterization}

Demographic questionnaire This will be used to describe self-reported medical and personal details like age, length of hospitalization, weight bearing status and medical services received post discharge.

\section{Outcome measures \\ Functional Independence Measure (FIM) [71]}

The aim of the FIM is to monitor the recovery of functional ability by people undergoing rehabilitation. The FIM is comprised of 18 parameters, each rated on a scale of 1-7 (range $=18-126)$ according to the degree of assistance required to perform a specific activity.

\section{2-item MOS Short-Form Health Status Survey [72], Hebrew version [73]}

This generic HR-QoL instrument focuses on functional status. The questionnaire includes 12 items taken directly from the SF-36 [74] which are used to calculate the Physical and Mental Component Summary. The first question measured by the SF-12 is an acceptable self-rated measure for general health [75].

\section{The Geriatric Depression Scale (GDS)-Short [76], Hebrew version [77]}

The scale consists of 15 items; each item has two possible answers (yes or no). The highest possible score is 15, which indicates the most severe depressive state.

The Canadian Occupational Performance Measure (COPM) [65] will be used for measuring performance and satisfaction with personally identified participation goals. Participants are asked to identify goals and then rate their performance and satisfaction with current status on a scale 
from 1 to 10, where 10 indicates optimal performance or satisfaction.

\section{The Zarit Caregiver Burden Interview (ZBI) [78]}

The ZBI includes 22 statements recorded in a 0-4 Likert scale (total score range 0 to 88 , where higher scores mean higher burden), which rates the subjective component of burden.

\section{Qualitative interview}

Semi-structured interviews will be conducted post intervention with the patients in the research groups $(n=60)$ and their caregivers.

$(n=60)$ to identify barriers and facilitators regarding the telerehabilitation and face-to-face programs.

\section{Data analysis}

Quantitative data will be analyzed using SPSS 21.0. Descriptive statistics will be obtained on the demographic, social and clinical pretest characteristics of subjects in the three groups. Differences in these variables between the intervention and control groups will be examined using either chi-square tests or analysis of variance. Feasibility in the research groups will be calculated according to the of participants who complete the trial.

Repeated-measures MANOVA will be used to test the effects of the group assignments (intervention vs. control), the effects of time (baseline, post-intervention, and 6-month follow-up), and the group-by-time interaction for measuring all the outcomes. Repeated-measures MANOVA is recommended as being straightforward, powerful, and appropriate to treatment outcome investigations [79]. In addition to providing a multivariate analysis of the effect of treatment on the combined changes in all measures and healthcare costs, this approach also provides a univariate analysis of the effect of treatment on each measure, thus allowing an investigation of which measures were most affected by treatment [79]. $p$-Values less than 0.05 are considered to be statistically significant in all comparisons.

All qualitative interviews (patients and caregivers) will be video/audio-recorded and transcribed. Conventional content analysis with constant comparative methods will be used for coding and analysis. All data will be collected anonymously, and access will be restricted to the investigators.

\section{Sample size calculation}

The sample size was based on RCT that compared the effectiveness of face-to-face home rehabilitation to conventional care after hip fracture of geriatric patients [64]. The home rehabilitation group showed a higher degree of recovery in self-care using the FIM, (calculated effect size: $d$
$=0.8$ ). The minimum sample size at the significance level of $5 \%$ (one-sided test) and power of $80 \%$ is 25 in each group. Estimated drop-out rate is $20 \%$; therefore, a maximum of 30 participants will be recruited for each group.

\section{Discussion}

This study has the potential to guide clinical practice toward innovative modes of rehabilitation care and enhance clinical management of hip fracture in older people. If efficacious, our results will provide a novel online delivery method that rehabilitation professionals can incorporate into their treatment program, which in turn will optimize outcomes. The proposed innovative telerehabilitation directly addresses one of the major issues of geriatric rehabilitative care - continuity. It is designed to support the weakest links in the chain of care transition [26]. It will assist in the transition from hospital to community dwelling, as well as maintaining the rehabilitation achievements. Since the hours of supervised therapy are limited, telerehabilitation will enable patients to expand significantly the hours that they practice therapeutic exercises [54], while promoting more effective methods of long-term rehabilitation and maintenance of a healthy lifestyle [80].

There are barriers to overcome relating to patients' personal restrictions, staff logistical issues of the systems to ensure the implementation of telerehabilitation more widely in older adults [81]. However, community-dwelling older people who have received a home telerehabilitation program found telerehabilitation convenient and motivating, coped well with the technology, and developed positive therapeutic relationships [82]. In addition, the current research will allow examination of the cost-effectiveness of telerehabilitation in a large cohort of patients after hip fracture. The availability of this service will enable reduction of the length of rehabilitation hospitalizations, and will be used as an alternative to conventional ambulatory rehabilitation, particularly for those patients in rural and peripheral areas. Furthermore, this type of service is compatible with the evolving models of self-management, in which clients are encouraged to take responsibility for managing their diseases or impairments [83].

To summarize, three major innovations are presented in our suggested protocol: First, this protocol outlines the justification for the first RCT study in telerehabilitation service for patients after hip fracture. Second, our proposed service is based on available, easy-to-use, inexpensive, simple technology [46], using off-the-shelf applications. Last, the focus is on individualized day-today function rather than exercising, as an individualized approach may be most effective due to the patient's unique characteristics [84]. 


\section{Abbreviations}

ADL: Activities of daily living; CO - OP: Cognitive Orientation to daily Occupational Performance; COPM: The Canadian Occupational Performance Measure; FIM: Functional Independence Measure; GDS: Geriatric Depression Scale; MOCA: Montreal Cognitive Assessment; QOL: Quality of life; RCT: Randomized controlled trial; SF- 12: 12-item MOS Short-Form Health Status Survey; ZBI: The Zarit Caregiver Burden Interview.

\section{Acknowledgments}

We would like to thank The Israel National Institute for Health Policy and Health Services Research (NIHP) for their financial support

\section{Funding}

This study is funded by The Israel National Institute for Health Policy and Health Services Research (NIHP) (\#2016/191/A).

\section{Availability of data and materials}

Only materials relating to the study protocol is reported in this paper. There is no raw data reported. Issues pertaining to this paper can be made to the corresponding author on reasonable request.

\section{Authors' contributions}

YG and YK, SK, MEE were responsible for the study conception and design. YG was responsible for contributing to the calculation of the sample size. TM was primarily responsible for drafting the manuscript with support from YG. SK, MEE, $\mathrm{TM}, \mathrm{YK}, \mathrm{ML}, \mathrm{IS}$ are responsible for ongoing study procedure, data management and analyses. YG is the trial sponsor, assumes responsibility writing of the reports and the decision to submit the report for publication. All authors critically reviewed the manuscript for content and style and approved the final version of the manuscript for submission

\section{Ethics approval and consent to participate}

This study received ethical approval from the Herzog Hospital institutional review Board (\# 292-15). Participants will receive information leaflets and give written informed consent.

\section{Consent for publication}

Not applicable.

\section{Competing interests}

The authors declare that they have no competing interests.

\section{Publisher's Note}

Springer Nature remains neutral with regard to jurisdictional claims in published maps and institutional affiliations.

\section{Author details}

ISchool of Occupational Therapy, Faculty of Medicine, Hadassah and the Hebrew University of Jerusalem, Mount Scopus, 91240 Jerusalem, Israel. ${ }^{2}$ Herzog Medical Center, Givat Shaul, P.O.Box 3900, 91035 Jerusalem, Israel. ${ }^{3}$ Hadassah University Hospital, P.O.Box 12000, 91120 Jerusalem, Israel.

Received: 21 December 2017 Accepted: 22 April 2019

Published online: 20 May 2019

\section{References}

1. Brauer CA, Coca-Perraillon M, Cutler DM, Rosen AB. Incidence and mortality of hip fractures in the United States. Jama. 2009;302(14):1573-9. https://doi. org/10.1001/jama.2009.1462.

2. Bertram M, Norman R, Kemp L, Vos T. Review of the long-term disability associated with hip fractures. Injury Prevention. 2011;17(6):365-70. https:// doi.org/10.1136/ip.2010.029579.

3. Taylor NF, Peiris CL, Kennedy G, Shields N. Walking tolerance of patients recovering from hip fracture: a phase I trial. Disabil Rehabil. 2016:1-9. https://doi.org/10.3109/09638288.2015.1107776.

4. Leal J, Gray AM, Prieto-Alhambra D, Arden NK, Cooper C, Javaid MK, et al. Impact of hip fracture on hospital care costs: a population-based study. Osteoporosis Int. 2016;27(2):549-58. https://doi.org/10.1007/s00198-015-3277-9.

5. Nguyen ND, Ahlborg HG, Center JR, Eisman JA, Nguyen TV. Residual lifetime risk of fractures in women and men. J Bone Mineral Res. 2007;22(6):781-8. https://doi.org/10.1359/jbmr.070315
6. Caeiro JR, Bartra A, Mesa-Ramos M, Etxebarria I, Montejo J, Carpintero P, et al. Burden of first osteoporotic hip fracture in Spain: a prospective, 12month, observational study. Calcif Tissue Int. 2016. https://doi.org/10.1007/ s00223-016-0193-8.

7. Bleibler F, Konnopka A, Benzinger P, Rapp K, Konig HH. The health burden and costs of incident fractures attributable to osteoporosis from 2010 to 2050 in Germany--a demographic simulation model. Osteoporosis Int. 2013; 24(3):835-47. https://doi.org/10.1007/s00198-012-2020-z.

8. Polinder S, Haagsma J, Panneman M, Scholten A, Brugmans M, Van Beeck E. The economic burden of injury: health care and productivity costs of injuries in the Netherlands. Accid Anal Prev. 2016;93:92-100. https://doi.org/ 10.1016/j.aap.2016.04.003.

9. Amling M, Oheim R, Barvencik F. A holistic hip fracture approach: individualized diagnosis and treatment after surgery. Eur J Trauma Emerg Surg. 2014;40(3):265-71. https://doi.org/10.1007/s00068-014-0374-y.

10. Ftouh S, Morga A, Swift C. Management of hip fracture in adults: summary of NICE guidance. BMJ: British Medical Journal (Overseas \& Retired Doctors Edition). 2011;342(7812):1-3.

11. Langford DP, Fleig L, Brown KC, Cho NJ, Frost M, Ledoyen M, et al. Back to the future - feasibility of recruitment and retention to patient education and telephone follow-up after hip fracture: a pilot randomized controlled trial. Patient Prefer Adherence. 2015;9:1343-51. https://doi.org/10.2147/ppa. s86922

12. Kanis JA, Oden A, Johnell O, De Laet C, Jonsson B, Oglesby AK. The components of excess mortality after hip fracture. Bone. 2003;32(5):468-73.

13. Orive M, Aguirre U, Garcia-Gutierrez S, Las Hayas C, Bilbao A, Gonzalez N, et al. Changes in health-related quality of life and activities of daily living after hip fracture because of a fall in elderly patients: a prospective cohort study. Int J Clin Pract. 2015;69(4):491-500. https://doi.org/10.1111/ijcp.12527.

14. Rasmussen B, Uhrenfeldt L. Establishing well-being after hip fracture: a systematic review and meta-synthesis. Disabil Rehabil. 2016:1-15. https://doi. org/10.3109/09638288.2016.1138552.

15. Mariconda M, Costa GG, Cerbasi S, Recano P, Orabona G, Gambacorta M, et al. Factors predicting mobility and the change in activities of daily living after hip fracture: a 1-year prospective cohort study. J Orthop Trauma. 2016; 30(2):71-7. https://doi.org/10.1097/bot.0000000000000448.

16. Ziden L, Wenestam CG, Hansson-Scherman M. A life-breaking event: early experiences of the consequences of a hip fracture for elderly people. Clin Rehabil. 2008;22(9):801-11. https://doi.org/10.1177/0269215508090204.

17. Karni S, Bentur N, Ratzon N. Participation and quality of life of cognitively impaired older women in Israel following hip fractures. Occup Ther Int. 2014;21(3):91-7. https://doi.org/10.1002/oti.1365.

18. Ponten JB, Krug E, van Baardewijk $L$, van der Linden EH, Haas R, Krijnen $P$, et al. Intensive rehabilitation in selected hip fracture patients may optimize care efficiency: a retrospective comparison study. J Rehabil Med. 2015;47(3): 278-81. https://doi.org/10.2340/16501977-1917.

19. Hernlund E, Svedbom A, Ivergard M, Compston J, Cooper C, Stenmark J, et al. Osteoporosis in the European Union: medical management, epidemiology and economic burden. A report prepared in collaboration with the International Osteoporosis Foundation (IOF) and the European Federation of Pharmaceutical Industry Associations (EFPIA). Archives Osteoporosis. 2013:8:136. https://doi.org/10.1007/s11657-013-0136-1.

20. Lin PC, Hung SH, Liao MH, Sheen SY, Jong SY. Care needs and level of care difficulty related to hip fractures in geriatric populations during the postdischarge transition period. J Nurs Res. 2006;14(4):251-60.

21. Leland NE, Gozalo P, Christian TJ, Bynum J, Mor V, Wetle TF, et al. An examination of the first 30 days after patients are discharged to the community from hip fracture Postacute care. Med Care. 2015;53(10):879-87. https://doi.org/10.1097/mlr.0000000000000419.

22. Zucker I, Laxer I, Rasooli I, Han S, Cohen A, Shohat T. Regional gaps in the provision of inpatient rehabilitation services for the elderly in Israel: results of a national survey. Israel J Health Policy Res. 2013;2:27. https://doi.org/10. 1186/2045-4015-2-27.

23. Hershkovitz A, Pulatov I, Brill S, Beloosesky Y. Can hip-fractured elderly patients maintain their rehabilitation achievements after 1 year? Disabil Rehabil. 2012;34(4):304-10. https://doi.org/10.3109/09638288.2011.606346.

24. Salpakoski A, Tormakangas T, Edgren J, Kallinen M, Sihvonen SE, Pesola M, et al. Effects of a multicomponent home-based physical rehabilitation program on mobility recovery after hip fracture: a randomized controlled trial. J Am Med Dir Assoc. 2014;15(5):361-8. https://doi.org/10.1016/j.jamda. 2013.12.083. 
25. Sims-Gould J, Byrne K, Hicks E, Khan K, Stolee P. Examining "success" in post-hip fracture care transitions: a strengths-based approach. J Interprofessional Care. 2012;26(3):205-11. https://doi.org/10.3109/13561820. 2011.645090.

26. McLeod J, Stolee P, Walker J, Heckman G. Measuring care transition quality for older patients with musculoskeletal disorders. Musculoskeletal Care. 2014;12(1):13-21. https://doi.org/10.1002/msc.1043.

27. Popejoy LL, Dorman Marek K, Scott-Cawiezell J. Patterns and problems associated with transitions after hip fracture in older adults. J Gerontol Nurs. 2013;39(9):43-52. https://doi.org/10.3928/00989134-20130620-01.

28. Ginsberg G, Adunsky A, Rasooly I. A cost-utility analysis of a comprehensive orthogeriatric care for hip fracture patients, compared with standard of care treatment. Hip Int. 2013;23(6):570-5. https://doi.org/10.5301/hipint.5000080.

29. Stolee P, Lim SN, Wilson L, Glenny C. Inpatient versus home-based rehabilitation for older adults with musculoskeletal disorders: a systematic review. Clin Rehabil. 2012;26(5):387-402. https://doi.org/10.1177/ 0269215511423279.

30. Zidén L, Kreuter M, Frändin K. Long-term effects of home rehabilitation after hip fracture-1-year follow-up of functioning, balance confidence, and healthrelated quality of life in elderly people. Disabil Rehabil. 2010;32(1):18-32.

31. Seitz DP, Gill SS, Austin PC, Bell CM, Anderson GM, Gruneir A, et al. Rehabilitation of older adults with dementia after hip fracture. J Am Geriatr Soc. 2016;64(1):47-54. https://doi.org/10.1111/jgs.13881.

32. Edgren J, Salpakoski A, Sihvonen SE, Portegijs E, Kallinen M, Arkela M, et al. Effects of a home-based physical rehabilitation program on physical disability after hip fracture: a randomized controlled trial. J Am Med Directors Assoc. 2015;16(4):350.e1-7. https://doi.org/10.1016/j.jamda.2014.12.015.

33. Hagsten B, Svensson O, Gardulf A. Health-related quality of life and selfreported ability concerning $A D L$ and IADL after hip fracture: a randomized trial. Acta Orthop. 2006;77(1):114-9.

34. Phelan EA, Williams B, Penninx BW, LoGerfo JP, Leveille SG. Activities of daily living function and disability in older adults in a randomized trial of the health enhancement program. J Gerontol A Biol Sci Med Sci. 2004;59(8):838-43.

35. Stuck AE, Egger M, Hammer A, Minder CE, Beck JC. Home visits to prevent nursing home admission and functional decline in elderly people: systematic review and meta-regression analysis. Jama. 2002; 287(8):1022-8.

36. Sanford JA, Griffiths PC, Richardson P, Hargraves K, Butterfield T, Hoenig H. The effects of in-home rehabilitation on task self-efficacy in mobility-impaired adults: a randomized clinical trial. J Am Geriatr Soc. 2006;54(11):1641-8.

37. Sanford JA, Hoenig H, Griffiths PC, Butterfield T, Richardson P, Hargraves K. A comparison of televideo and traditional in-home rehabilitation in mobility impaired older adults. Phys Occup Therapy Geriatrics. 2007;25(3):1-18.

38. Tousignant M, Boissy P, Corriveau H, Moffet $\mathrm{H}$. In home telerehabilitation for older adults after discharge from an acute hospital or rehabilitation unit: a proof-ofconcept study and costs estimation. Disabil Rehabil Assist Technol. 2006;1 (4):209-16.

39. Gaikwad R, Warren J. The role of home-based information and communications technology interventions in chronic disease management: a systematic literature review. Health Informatics J. 2009;15(2):122-46. https://doi.org/10.1177/1460458209102973.

40. Bashshur RL, Shannon GW, Smith BR, Alverson DC, Antoniotti N, Barsan WG, et al. The empirical foundations of telemedicine interventions for chronic disease management. Telemed J e-health. 2014;20(9):769-800. https://doi. org/10.1089/tmj.2014.9981.

41. Blozik E, Wildeisen IE, Fueglistaler P, von Overbeck J. Telemedicine can help to ensure that patients receive timely medical care. J Telemed Telecare. 2012;18(2):119-21. https://doi.org/10.1258/jtt.2011.110812.

42. Koutras C, Bitsaki M, Koutras G, Nikolaou C, Heep H. Socioeconomic impact of e-health services in major joint replacement: a scoping review. Technol Health Care. 2015;23(6):809-17. https://doi.org/10.3233/thc-151036.

43. Tindall LR, Huebner RA. The impact of an application of telerehabilitation technology on caregiver burden. Int J Telerehabilitation. 2009;1(1):3-8.

44. Anton D, Nelson M, Russell T, Goni A, Illarramendi A. Validation of a Kinectbased telerehabilitation system with total hip replacement patients. J Telemed Telecare. 2015. https://doi.org/10.1177/1357633×15590019.

45. Brennan DM, Mawson S, Brownsell S. Telerehabilitation: enabling the remote delivery of healthcare, rehabilitation, and self management. Stud Health Technol Inform. 2009;145:231-48.

46. WFOT. World federation of occupational therapists' position statement on telehealth. Int J Telerehabilitation. 2014;6(1):37-9. https://doi.org/10.5195/ijt. 2014.6153.
47. Vuolio S, Winblad I, Ohinmaa A, Haukipuro K. Videoconferencing for orthopaedic outpatients: one-year follow-up. J Telemed Telecare. 2003;9(1):8-11.

48. Hoffmann T, Russell T. Pre-admission orthopaedic occupational therapy home visits conducted using the internet. J Telemed Telecare. 2008;14(2): 83-7. https://doi.org/10.1258/jtt.2007.070808.

49. Cha E, Castro HK, Provance P, Finkelstein J. Acceptance of home telemanagement is high in patients with multiple sclerosis. AMIA Annual Symp Proc. 2007:893:893.

50. Finkelstein J, Jeong IC. Feasibility of interactive biking exercise system for telemanagement in elderly. Stud Health Technol Inform. 2013;192:642-6.

51. Bedra M, Finkelstein J. Feasibility of post-acute hip fracture telerehabilitation in older adults. Stud Health Technol Informatics. 2015;210:469-73.

52. Crotty M, Killington M, van den Berg M, Morris C, Taylor A, Carati C. Telerehabilitation for older people using off-the-shelf applications: acceptability and feasibility. J Telemed Telecare. 2014;20(7):370-6. https://doi.org/10.1177/1357633×14552382.

53. Llorens R, Noe E, Colomer C, Alcaniz M. Effectiveness, usability, and costbenefit of a virtual reality-based telerehabilitation program for balance recovery after stroke: a randomized controlled trial. Archives Phys Med Rehabil. 2015;96(3):418-25.e2. https://doi.org/10.1016/j.apmr.2014.10.019.

54. Sugarman H, Dayan E, Weisel-Eichler A, Tiran J. The Jerusalem TeleRehabilitation system, a new low-cost, haptic rehabilitation approach. Cyberpsychol Behavior. 2006;9(2):178-82. https://doi.org/10.1089/cpb.2006.9.178.

55. Fusco F, Turchetti G. Telerehabilitation after total knee replacement in Italy: cost-effectiveness and cost-utility analysis of a mixed telerehabilitationstandard rehabilitation programme compared with usual care. BMJ Open. 2016;6(5):e009964. https://doi.org/10.1136/bmjopen-2015-009964.

56. Tousignant M, Moffet $H$, Nadeau S, Merette C, Boissy P, Corriveau H, et al. Cost analysis of in-home telerehabilitation for post-knee arthroplasty. J Med Internet Res. 2015;17(3):e83. https://doi.org/10.2196/jmir.3844.

57. Schulz KF, Altman DG, Moher D. CONSORT 2010 statement: updated guidelines for reporting parallel group randomised trials. BMC Med. 2010;8(1):18.

58. WHO. ICD-10: International Statistical Classification of Diseases and Related Health Problems. Geneva: World Health Organization; 2010.

59. Nasreddine ZS, Phillips NA, Bedirian V, Charbonneau S, Whitehead V, Collin I, et al. The Montreal cognitive assessment, MoCA: a brief screening tool for mild cognitive impairment. J Am Geriatr Soc. 2005;53(4):695-9. https://doi. org/10.1111/j.1532-5415.2005.53221.x.

60. Polatajko $\mathrm{H}$, Mandich A. Enabling occupation in children: the cognitive orientation to daily occupational performance (CO-OP) approach. Ottawa: CA CAOT; 2004

61. Polatajko HJ, McEwen SE, Ryan JD, Baum CM. Pilot randomized controlled trial investigating cognitive strategy use to improve goal performance after stroke. Am J Occup Ther. 2012;66(1):104-9.

62. Wolf TJ, Polatajko H, Baum C, Rios J, Cirone D, Doherty M, et al. Combined Cognitive-Strategy and Task-Specific Training Affects Cognition and UpperExtremity Function in Subacute Stroke: An Exploratory Randomized Controlled Trial. Am J Occup Ther. 2016;70(2):7002290010p1-p10. https://doi.org/10.5014/ ajot.2016.017293

63. Scammell EM, Bates SV, Houldin A, Polatajko HJ. The Cognitive Orientation to daily Occupational Performance (CO-OP): A scoping review: L'approche CO-OP (Cognitive Orientation to daily Occupational Performance) : examen de la portee. Can J Occup Ther. 2016;83(4):216-25. https://doi.org/10.1177/0008417416651277.

64. Ziden L, Frandin K, Kreuter M. Home rehabilitation after hip fracture. A randomized controlled study on balance confidence, physical function and everyday activities. Clin Rehabil. 2008;22(12):1019-33. https:/doi.org/10.1177/ 0269215508096183

65. Jackson JP, Schkade JK. Occupational adaptation model versus biomechanicalrehabilitation model in the treatment of patients with hip fractures. Am J Occup Ther. 2001;55(5):531-7.

66. McEwen S, Polatajko H, Baum C, Rios J, Cirone D, Doherty M, et al. Combined cognitive-strategy and task-specific training improve transfer to untrained activities in subacute stroke: an exploratory randomized controlled trial. Neurorehabil Neural Repair. 2014. https://doi.org/10.1177/ 1545968314558602.

67. McEwen SE, Polatajko HJ, Huijbregts MP, Ryan JD. Exploring a cognitive-based treatment approach to improve motor-based skill performance in chronic stroke: results of three single case experiments. Brain Inj. 2009;23(13-14):1041-53. https:// doi.org/10.3109/02699050903421107.

68. McEwen SE, Polatajko HJ, Huijbregts MP, Ryan JD. Inter-task transfer of meaningful, functional skills following a cognitive-based treatment: results of three multiple baseline design experiments in adults with chronic stroke. 
Neuropsychol Rehabil. 2010;20(4):541-61. https://doi.org/10.1080/ 09602011003638194.

69. Ng EM, Polatajko HJ, Marziali E, Hunt A, Dawson DR. Telerehabilitation for addressing executive dysfunction after traumatic brain injury. Brain Inj. 2013; 27(5):548-64. https://doi.org/10.3109/02699052.2013.766927.

70. Freedland KE, Mohr DC, Davidson KW, Schwartz JE. Usual and unusual care: existing practice control groups in randomized controlled trials of behavioral interventions. Psychosom Med. 2011;73(4):323.

71. Keith RA, Granger CV, Hamilton BB, Sherwin FS. The functional independence measure: a new tool for rehabilitation. Adv Clin Rehabil. 1987;1:6-18.

72. Ware JE Jr, Kosinski M, Keller SD. A 12-item short-form health survey: construction of scales and preliminary tests of reliability and validity. Med Care. 1996;34(3):220-33.

73. Amir M, Lewin-Epstein N, Becker G, Buskila D. Psychometric properties of the SF-12 (Hebrew version) in a primary care population in Israel. Med Care. 2002:40(10):918-28. https://doi.org/10.1097/01.mlr.0000027359.33761.fa.

74. Ware JE Jr, Sherbourne CD. The MOS 36-item short-form health survey (SF-36). I. Conceptual framework and item selection. Med Care. 1992;30(6):473-83.

75. Chamberlain AM, McNallan SM, Dunlay SM, Spertus JA, Redfield MM, Moser DK, et al. Physical health status measures predict all-cause mortality in patients with heart failure. Circulation Heart failure. 2013;6(4):669-75. https:// doi.org/10.1161/circheartfailure.112.000291

76. Yesavage JA, Brink TL, Rose TL, Lum O, Huang V, Adey M, et al. Development and validation of a geriatric depression screening scale: a preliminary report. J Psychiatr Res. 1982;17(1):37-49.

77. Zalsman G, Aizenberg D, Sigler M, Nahshoni E, Weizman A. Geriatric depression scale-short form-validity and reliability of the Hebrew version. Clin Gerontol. 1998;18(3):3-9.

78. Zarit S, Orr NK, Zarit JM. The hidden victims of Alzheimer's disease: families under stress. New York: NYU Press; 1985.

79. O'Brien RG, Kaiser MK. MANOVA method for analyzing repeated measures designs: an extensive primer. Psychol Bull. 1985;97(2):316.

80. Korman M, Weiss P, Kizony R. Living labs: overview of ecological approaches for health promotion and rehabilitation. Disabil Rehabil. 2015:1-7.

81. Peel NM, Russell TG, Gray LC. Feasibility of using an in-home video conferencing system in geriatric rehabilitation. J Rehabil Med. 2011;43(4): 364-6. https://doi.org/10.2340/16501977-0675.

82. Shulver W, Killington M, Morris C, Crotty M. 'Well, if the kids can do it, I can do it': older rehabilitation patients' experiences of telerehabilitation. Health expectations : an international journal of public participation in health care and health policy. 2016. doi:https://doi.org/10.1111/hex.12443.

83. Kizony R, Harel S, Elion O, Weiss P, Feldman Y, Shani M et al., editors. Telerehabilitation service delivery: Journey from prototype to robust in-home use. Virtual Rehabilitation Proceedings (ICVR), 2015 International Conference on; 2015: IEEE.

84. Kessler DE, Egan MY, Dubouloz CJ, Graham FP, McEwen SE. Occupational performance coaching for stroke survivors: a pilot randomized controlled trial protocol. Can J Occup Ther. 2014;81(5):279-88,

Ready to submit your research? Choose BMC and benefit from:

- fast, convenient online submission

- thorough peer review by experienced researchers in your field

- rapid publication on acceptance

- support for research data, including large and complex data types

- gold Open Access which fosters wider collaboration and increased citations

- maximum visibility for your research: over $100 \mathrm{M}$ website views per year

At BMC, research is always in progress.

Learn more biomedcentral.com/submissions 